\title{
Design of an Automated Grain Storage Chamber Based on Wireless Sensor Network
}

\author{
Pankaj Sharma, Paramita Guha*, Sunita Mishra
}

\begin{abstract}
The main objective of this paper is to design a monitoring and controlling system for storage of wheat grains. The temperature, humidity, $\mathrm{CO}_{2}$ and $\mathrm{O}_{2}$ gas levels are the main controlling parameters. Sensors are placed inside the chamber to monitor them and the data is analyzed using wireless communication devices. The data of all the sensors is sent to a wireless zigbee coordinator which is again connected to a microcontroller PIC16F877A. Depending upon the output of the sensor, an actuator operates. The design and implementation of this process has been done using PIC microcontroller, Zigbee \& LABVIEW software. This paper provides details of sensing, data acquisition, and controlling the storage parameters.
\end{abstract}

Keywords-Wireless sensor network, PIC 16F877A, Zigbee, LabVIEW, Sensor, Actuator

\section{Introduction}

There is a vast improvement in communication system in this century. Depending upon the operations, communication systems can be classified into two different categories, e.g. wired and wireless. Wired technology is based on collection of data using sensors and the data is transmitted using a large number of cables. Although this technique is quite simple, the main disadvantages are that more interference, lighting strikes, corrosions at joints along with the high maintenance costs [1], [2]. These drawbacks can mostly be overcome with the use of wireless network. Here the information from the source to the destination passes without wires. The distance between the two communication points may be short, medium or long. Short range data transfer can be done with the help of infrared region of electromagnetic spectrum whereas others can be done through wi-fi, zigbee, micro and radio frequency waves etc. In this paper, wireless communication technique based on zigbee technology [3], [4] is used for the design of an automated grain storage chamber.

Pankaj Sharma, Senior Project Fellow

CSIR-Central Scientific Instruments Organisation Chandigarh, India

\section{Paramita Guha, Scientist}

CSIR-Central Scientific Instruments Organisation

Chandigarh, India

$\mathrm{F}$

*Corresponding Author

Sunita Mishra, Primcipal Scientist

CSIR-Central Scientific Instruments Organisation

Chandigarh, India
Grains are produced in surplus hence, it is necessary that it should be properly stored properly. Hence, it should be stored properly. It has been observed that the food values of grains are mainly affected by the changes in temperature and humidity [5] [6]. As the temperature and humidity increase to a certain level, there is a growth of fungi, insects and microbes. These also detoriate the food value along with the shelf life of grains. Also, $\mathrm{CO}_{2}$ and $\mathrm{O}_{2}$ levels play important roles in the proper storage of grains [7]. Hence, monitoring and controlling of these parameters are quite important.

There are a very few research work is reported on wireless communication for grain storage chamber [8] [9]. The basic methodology is that sensors collect the data inside from the chamber and they send the data to the control center wirelessly via transreceivers. However, this technique still involves multicable and multijoints. In [10], authors have developed a chamber to monitor the inside temperature wirelessly. However, measurement of other parameters like, humidity, $\mathrm{CO}_{2}, \mathrm{O}_{2}$ gas levels are not mentioned. Although measurement of temperature and humidity of a storage chamber is given in [11], the control of them are not discussed. In this paper, an automated grain storage chamber is designed in which temperature, humidity, $\mathrm{CO}_{2}, \mathrm{O}_{2}$ gas levels are monitored and controlled wirelessly. The rest of the paper is organized in the following way. Following the introductory section, design of the chamber in given in Section II and the implementation of the proposed methodology is given in the next section. Results and discussions are given in Section IV and conclusion obtained from this paper is given in the subsequent section.

\section{Design}

For the present paper, a typical storage system is considered and its 2-dimensional view is presented in Figure 1 . The storage system is placed on the ground, hence the lower boundary is subjected to only convective heat transfer but the other boundaries will have both convective as well as radiative heat transfers. In this chamber, several sensors are placed to monitor the controlling parameters. The sensor probes are made of stainless steel and their compensation coefficients [12] are given in Table 1. 
Proc. of The Third Intl. Conf. on Advances in Computing, Control and Networking - ACCN 2015. Copyright $(\odot$ Institute of Research Engineers and Doctors, USA .All rights reserved.

ISBN: 978-1-63248-082-8 doi: 10.15224/ 978-1-63248-082-8-04

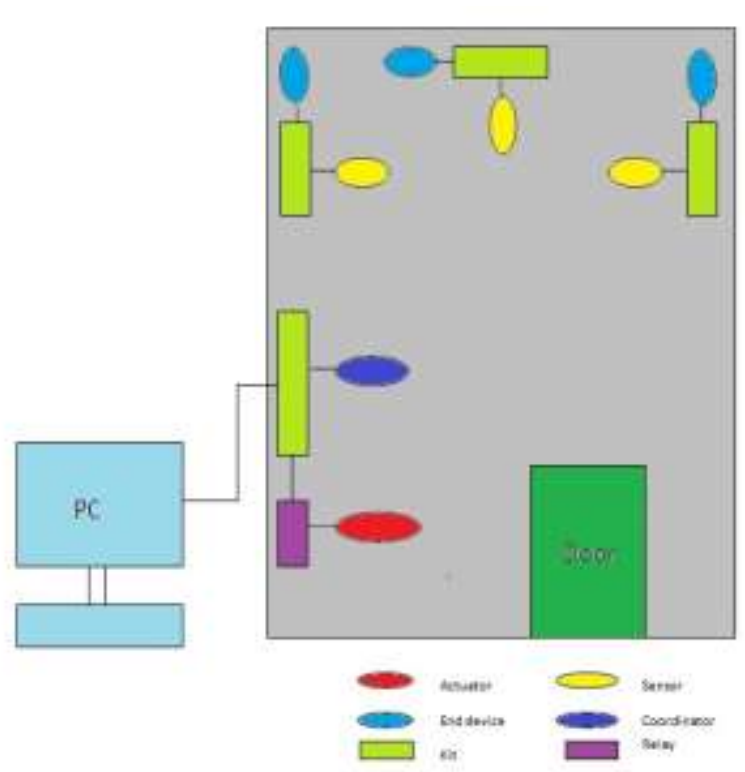

Figure 1. The schematic view of a storage system

\begin{tabular}{|c|c|c|c|c|}
\hline \multirow{2}{*}{$\begin{array}{c}\text { Sensor } \\
\text { Readout }\end{array}$} & \multicolumn{3}{|c|}{ Coefficients } & \multirow[t]{2}{*}{ Compensation } \\
\hline & $R 1$ & $R 2$ & R3 & \\
\hline Temperature & $1.02 \mathrm{E}-3$ & $2.22 \mathrm{E}-4$ & $1.33 \mathrm{E}-7$ & Non Linear \\
\hline Humidity & -0.258 & 0.304 & & Linear \\
\hline $\mathrm{CO} 2$ & 0 & 25000 & & Linear \\
\hline $\mathrm{O} 2$ & 0 & 0.065 & & Linear \\
\hline
\end{tabular}

The sensors are connected to corresponding zigbee enddevices thorough microcontroller kits. All the end-devices are connected to a main microcontroller $(\mu \mathrm{C})$ kit (PIC 16F877A) through a single coordinator. The $\mu \mathrm{C}$ is connected to a relay card. The controller senses the reading of sensors and depending upon the threshold values, the controller sends signals to the relay card and an actuator will respond. The actuators include air conditioner, heater, dehumidifier, humidifier, gas cylinders etc. In other words, as the PIC16F877A finds the temperature of the chamber is more than the threshold temperature value, the relay will actuate the air-conditioner and so on. The block diagram of the wireless sensing model is given in Figure 2.

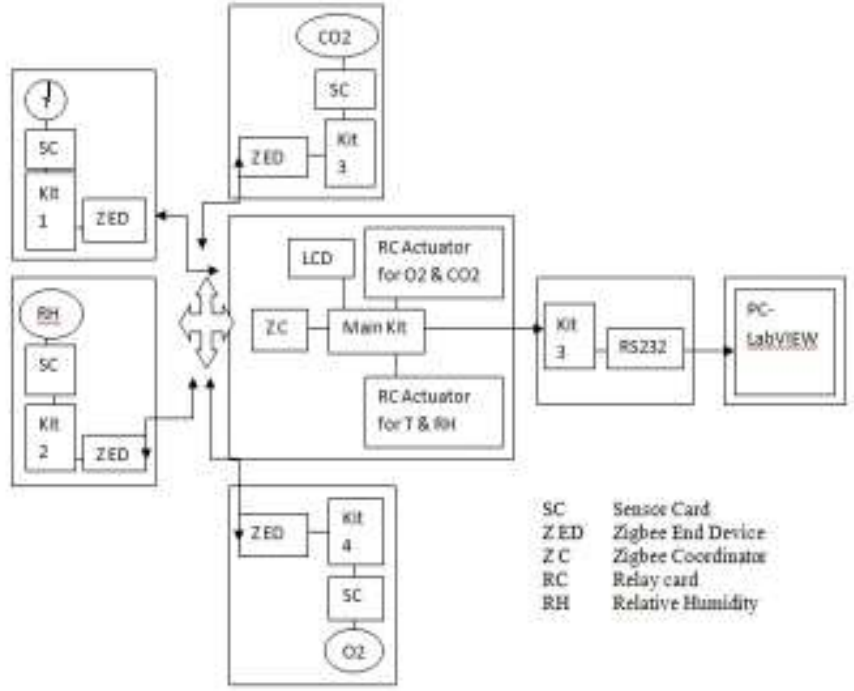

Figure 2. Block diagram of the wireless sensor network model of storage chamber

\section{Implementation}

As discussed in the previous section, the PIC $\mu \mathrm{C}$ will send signals to the actuators through relay cards. The protocol of the operation of each sensor is developed. Flow chart of the protocol to sense temperature is shown in Figure 3. After getting the data from sensors, end device will try to connect a zigbee coordinator. The coordinator will convert the analog voltage into its equivalent degree Celsius value and that value will be stored in another $\mu \mathrm{C}$ kit for further processing. This technique has been implemented for other parameters as well. After accumulating all the data, the coordinator processes them and one part is sent to the relay card and the other part is transmitted to a PC for the display at LCD. This operation is shown in Figure 4.

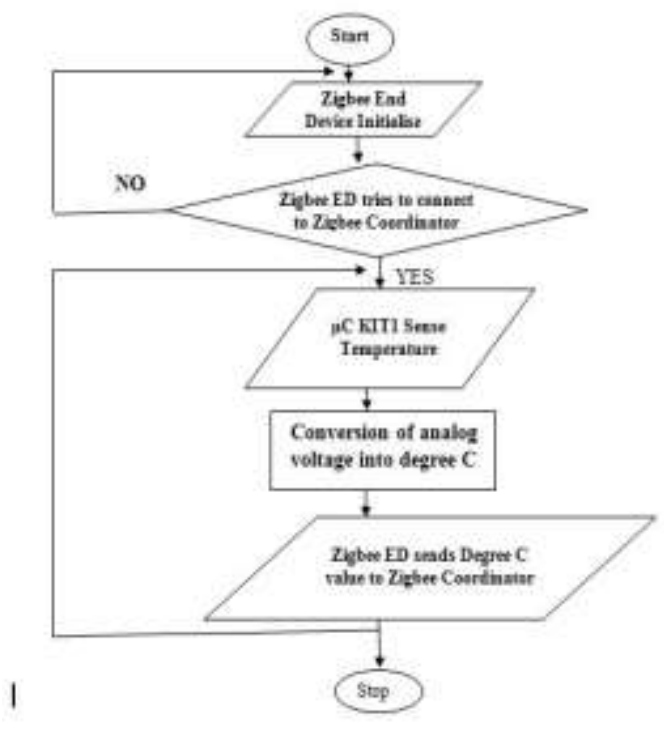

Figure 3. Flow chart for protocol of temperature sensor operation 
Proc. of The Third Intl. Conf. on Advances in Computing, Control and Networking - ACCN 2015.

Copyright $(\odot$ Institute of Research Engineers and Doctors, USA .All rights reserved.

ISBN: 978-1-63248-082-8 doi: 10.15224/ 978-1-63248-082-8-04

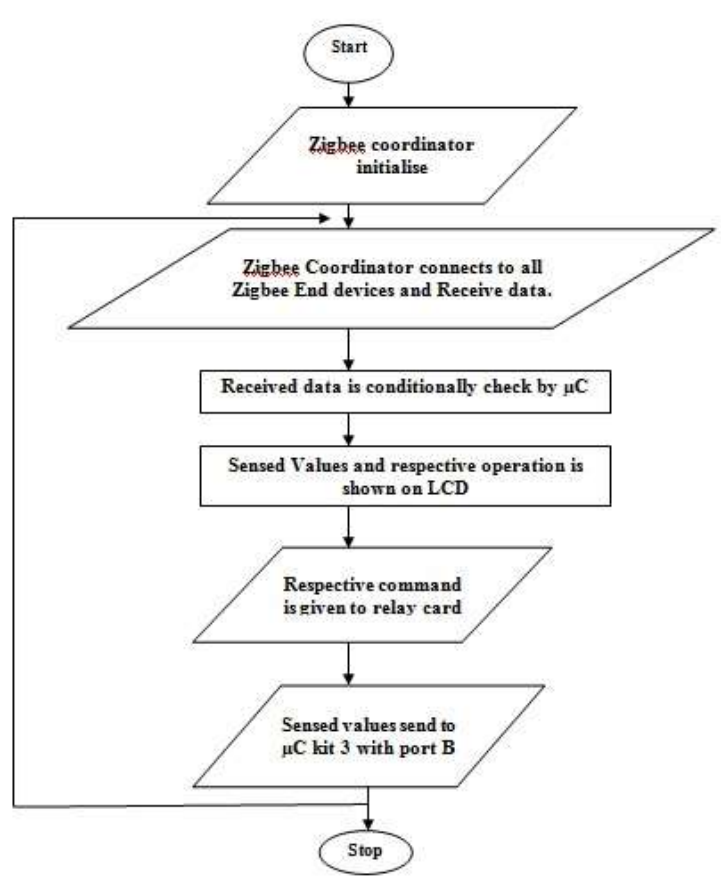

Figure 4. Flow chart for protocol of operation of main kit

In the PC, LabVIEW software is used to receive the data coming from $\mu \mathrm{C}$ kit with the help of VISA protocol. Data is further processed and conditionally checked. The values of the sensor and respective actuator operation needed are displayed on the front panel of the LabVIEW. The values are collected with the help of Write to measurement file icon which further get stored at the user given location for further processing.

\section{Results \& Discussion}

As discussed in section III, the protocols shown in Figure 3 are implemented for all the sensors and the output data is recorded at different environmental conditions. Two platforms are used for the display of output, viz., LabVIEW front panel and the LCD. Here, operation of respective actuator is shown using glowing LEDs. It has been observed from the literature that the wheat grains are generally stored at $20-25^{\circ} \mathrm{C}$ temperature with humidity remaining at $20-30 \%$. Also, the carbon dioxide and oxygen levels should be maintained approximately around 1500-2000 ppm and 20-30 ppm respectively. In the protocols, operating conditions of actuators are given based on these ranges. In Figure 5, it is shown that the value of temperature is around $28^{\circ} \mathrm{C}$ which is more than the upper threshold, hence the actuator airconditioner is activated automatically and the corresponding LED glows. Also, as the value of humidity is more than its upper threshold value (30\%), the dehumidifier is switched ON. Similarly, in Figure 6, another case study is shown where the output of $\mathrm{O}_{2}$ sensor is $16 \mathrm{ppm}$ which is less than the lower set value of controller, hence the valve of $\mathrm{O}_{2}$ cylinder is opened which is shown by glowing of corresponding LED along with the $\mathrm{AC} \mathrm{ON}$. The value of $\mathrm{CO}_{2}$ inside the chamber for an instant is measured as $2100 \mathrm{ppm}$, as this value more than the required upper threshold value, the exhaust fan is switched ON automatically which is shown in Figure 7. Another case is shown in Figure 8 where all the sensor readings (i.e. temperature, humidity, $\mathrm{CO}_{2}, \mathrm{O}_{2}$ ) are within the range. Hence, no actuator operation is required and the environmental parameters are maintained within their respective ranges.

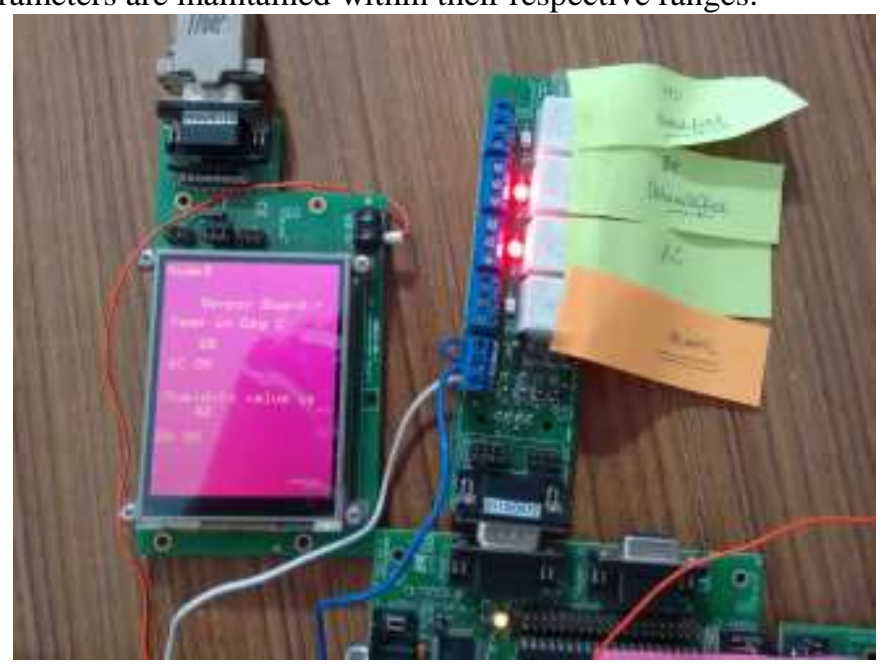

Figure 5: Operation of actuators AC \& dehumidifier

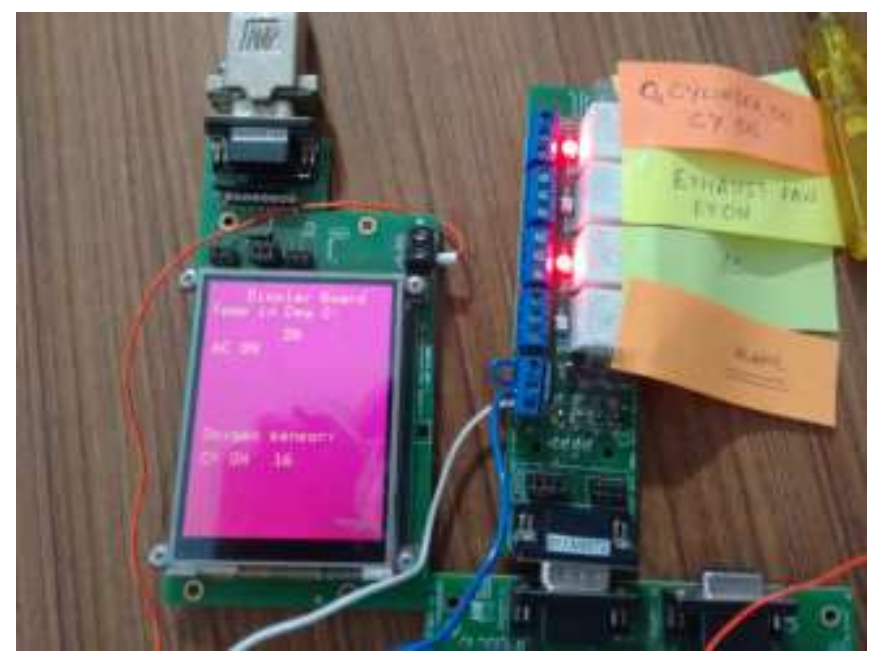

Figure 6. Operation of actuators AC \& oxygen cylinder

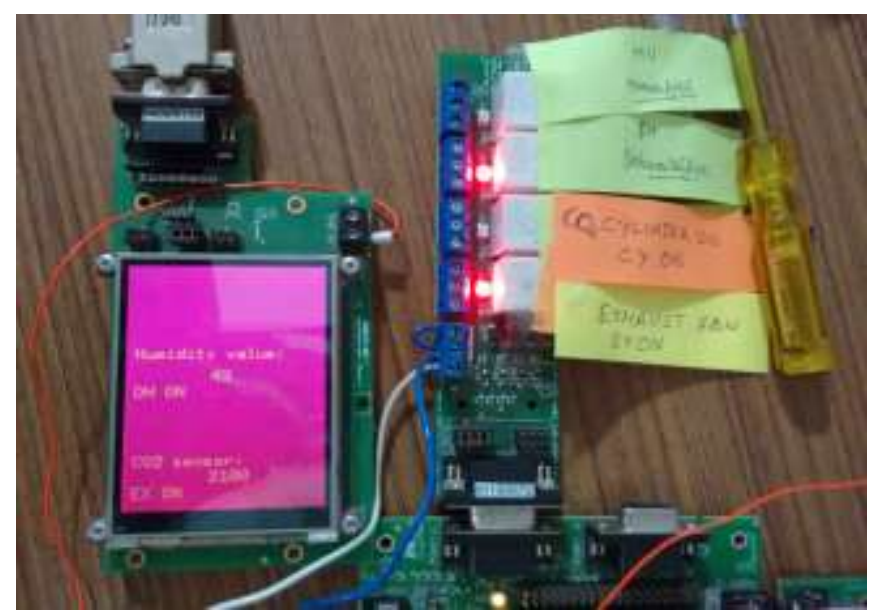

Figure 7. Operation of actuators Dehumidifier \& exhaust fan; 
Proc. of The Third Intl. Conf. on Advances in Computing, Control and Networking - ACCN 2015.

Copyright $(\odot$ Institute of Research Engineers and Doctors, USA .All rights reserved.

ISBN: 978-1-63248-082-8 doi: 10.15224/ 978-1-63248-082-8-04



Figure 8. No actuator operation

\section{v. Conclusion}

In this paper, a scheme for designing an automated grain storage chamber is presented. The sensors are placed inside the chamber to measure the environmental parameters. The data is sent to the controllers through wireless zigbee network. Depending upon the signal, the corresponding actuator is operated and the parameters of the chamber are maintained constant. The considered wireless network has several advantages over the conventional wired network like, low cost, miniaturized design, low energy consumption etc. Hence, the proposed model has a wide application prospect.

\section{References}

[1] A. Huynh, "Study of Wired and Wireless Data Transmissions", PhD thesis, Linköping University, 2010.

[2] R. Bansal, V. Gupta, and R. Malhotra, "Performance analysis of wired and wireless lan using soft computing techniques- A review", 2010.

[3] F. Zhang, H. Zhou, and X. Zhou, "A routing algorithm for zigbee network based on dynamic energy consumption decisive path", Int. Conf. on Computational Intelligence and Natural Computing, pp. 429 432, 2009.

[4] ZigBee Alliance, "ZigBee specification: ZigBee Document 053474r17," 17 Jan. 2008.

[5] D. W. Sun, and J. L. Woods, "Low temperature moisture transfer characteristics of wheat in thin-layers", American Society of Agricultural and Biological Engineers, vol. 37, pp. 1919-1926, 1994.

[6] C. Jia, "Study of heat and mass transfer inside grain kernel and temperature pattern in grain storage bin", $\mathrm{PhD}$ Thesis, Beijing Agricultural Engineering University, China, 1995.

[7] F. Lessard, D. Just, P. Barrieu, J. Torch, P. Raymond, and P. Saglio, "Effect of modified atmosphere storage on wheat seed germination vigour and on physiological criteria of the ageing process", Proc. of 6th Int. Working Conf. on stored product protection, pp. 695-700, 2012.

[8] W. Keqi and Z. Bin, "Research on Wireless Cyclic Test and Management System of Grain Information in Barns ," IEEE International Conference on Mechatronics and Automation (ICMA 2007), pp. 2231-2235, Aug. 2007

[9] L. Ze, H. Jiwei, and Z. Shuijing, "Design and Application of Distributive Wireless Monitoring System for Grain Status ," Electronic Engineer, no. 7, pp. 71-73, 2008

[10] L. Hui-li, Z. Ren-cheng, and M. Si-wen, "Design of Temperature Detection System for Grain Storag e Based on Wireless Sensors Network," Journal of Agricultural Mechanization Research , no.9, pp. 99-102, 2006.

[11] J. Jia, J. Kuang, Z. He, and Y. Mu, "Design of Monitor System for Grain Depots Based on Wireless Sensor Network", Proceedings of the 2009 IEEE International Conference on Mechatronics and Automation, pp. 2318-2323, 2009.

[12] http://www.vernier.com/products/sensors/

\section{Acknowledgment}

This paper is based on the work supported by CSIR, India under the project PSC0103-02. Authors are grateful and wish to express appreciation to many colleagues for discussion and their valuable inputs.

About Author (s):

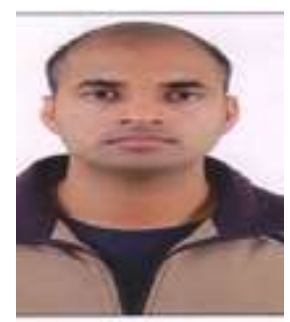

Pankaj Sharma has graduated in Electronics and communication in 2009 from SKIET Kurukshetra, Kurukshetra University, India. He has done his M. Tech in Electronics and communication in 2015 from PCET, Jalandhar, India. He is presently working as Senior Project Fellow at CSIRCSIO Chandigarh. His area of interests includes embedded System, wireless sensor networks and instrumentation.

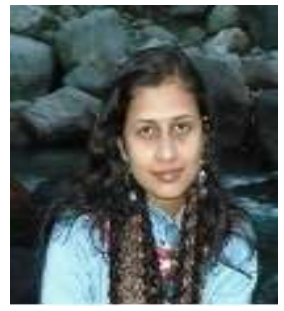

Paramita Guha graduated from Jalpaiguri Government Engineering College, India in 2001. She received her master's degree from Bengal Engineering College (Deemed University), India in 2003 and Ph.D. degree from Indian Institute of Technology (IIT), Delhi, India in 2012, both in electrical engineering. She is presently working as a scientist in CSIR-Central Scientific Instruments Organisation, Chandigarh, India. She has published several papers in international journal and conferences. Her research interests include distributed parameter systems, coupled systems, modeling and simulation, model reduction and control theory 
Proc. of The Third Intl. Conf. on Advances in Computing, Control and Networking - ACCN 2015.

Copyright $(\odot$ Institute of Research Engineers and Doctors, USA .All rights reserved.

ISBN: 978-1-63248-082-8 doi: 10.15224/ 978-1-63248-082-8-04

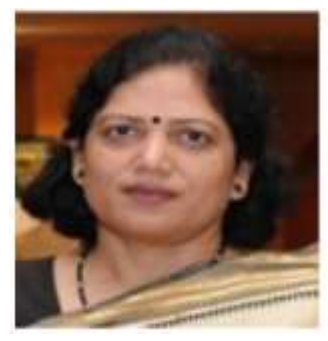

Sunita Mishra did her graduation and postgraduation in physics in the year 1983 and 1985, respectively from Allahabad University, Allahabad. She obtained her Ph.D. degree in physicselectronics engineering from Institute of Technology, Banaras Hindu University, Varanasi in 1993. At present she is working as a principal scientist in CSIR-Central Scientific Instruments Organisation, Chandigarh. She has completed projects in the area of agricultural electronics, gas sensors, semiconductor devices and NIR spectroscopy. She has published about 25 papers in journals and conference proceedings. She is life member of semiconductor society of India, Solar Energy Society of India and Material Research Society of India and the fellow of the Institution of Electronics and Telecommunication Engineers (IETE). Her research interests include sensors, semiconductor devices, and NIR spectroscopy. 